0038-1098(94)00833-7

\title{
MAGNETIC STRUCTURE OF SUPERLATTICES WITH BIQUADRATIC INTERLAYER EXCHANGE COUPLING
}

\author{
A.S. Carriço \\ Universidade Federal do Rio Grande de Norte, Departamento de Fisica/CCE 59072 Natal-RN, Brasil \\ and \\ D.R. Tilley* and R.E. Camley ${ }^{\dagger}$ \\ Department of Physics, University of Essex, Colchester CO4 3SQ, UK \\ (Received 3 August 1994; accepted in revised form 27 October 1994 by R. T. Phillips)
}

\begin{abstract}
We calculate the magnetic structure of an infinitely extended magnetic superlattice in the presence of interlayer biquadratic exchange coupling and a 4-fold in-plane anisotropy. An external magnetic field is also applied in the plane of the layers. We consider ground state configurations with unit cells involving 1, 2, or 4 magnetic layers. At low fields a two-layer unit cell structure is found to be the ground state. At higher fields the ground state has the spins in all layers aligned so the unit cell is one layer. Analytic expressions for the magnetic equilibrium structures are obtained in the small field limit, as are expressions for the critical fields for a transition from one state to another. Numerical results are presented for magnetic fields of arbitrary size.
\end{abstract}

THE DISCOVERY of biquadratic coupling between the $\mathrm{Fe}$ layers in $\mathrm{Fe} / \mathrm{Cr} / \mathrm{Fe}$ sandwiches at specific $\mathrm{Cr}$ thicknesses [1] has aroused considerable interest. The equilibrium structure of the $\mathrm{Fe} / \mathrm{Cr} / \mathrm{Fe}$ sandwich in the presence of biquadratic exchange is characterized experimentally $[2,3]$ by the spins in the two films differing in orientation by $90^{\circ}$ in the absence of an applied magnetic field. There have also been experimental studies on multilayer structures with different components such as $\mathrm{Fe} / \mathrm{Al} / \mathrm{Fe}$ [4], $\mathrm{NiFe} / \mathrm{Ag}$ [5] and $\mathrm{Co} / \mathrm{Cu}$ [6]. Theoretical spin configurations for the sandwich structure have been obtained by a number of groups [1,7], and a variety of physical mechanisms have been suggested for the biquadratic coupling [8]. Most of these studies have considered systems where the Zeeman, anisotropy, bilinear exchange as well as biquadratic exchange are all included. This leads to complex phase diagrams.

\footnotetext{
* After October 1994: School of Physics, Universiti Sains Malaysia, 11800 USAM Penang, Malaysia.

† Permanent address: Dept. of Physics, University of Colorado at Colorado Springs, Colorado Springs, CO 80933-7150, USA.
}

In contrast to the previous work, in this note we consider a superlattice of ferromagnetic (Fe) layers magnetized in plane and with biquadratic coupling as the only interaction between layers. This model allows us to carefully investigate the influence of an external magnetic field which is oriented in an arbitrary direction within the plane of the layers. Furthermore, we also investigate structures which are not available in the sandwich configuration. For example here we study systems with unit cells consisting of 1,2 , or 4 layers.

The $y$ axis is taken normal to the layers. With the magnetization in layer $n$ making an angle $\theta_{n}$ with the $x$ axis, the biquadratic coupling Hamiltonian is

$$
H_{\mathrm{BQ}}=\sum B \cos ^{2}\left(\theta_{n}-\theta_{n-1}\right)
$$

where the surn is over $N$ layers and periodic boundary conditions are used. We include cubic in-plane anisotropy and the Zeeman energy with a static field at angle $\phi$ from the $x$ axis:

$$
\begin{aligned}
& H_{A}=-\sum K\left(\cos ^{4} \theta_{n}+\sin ^{4} \theta_{n}\right) \\
& H_{Z}=-H \sum \cos \left(\theta_{n}-\phi\right)
\end{aligned}
$$


With $K$ positive, as we assume, $H_{A}$ favours alignment of $\theta_{n}$ along the $x$ or $z$ axis. The complete Hamiltonian is

$H=H_{\mathrm{BQ}}+H_{A}+H_{Z}$.

We first discuss qualitatively the equilibrium configurations of this Hamiltonian for infinite superlattices. If there is no applied field, then the lowest eigenvalue of $H_{\mathrm{BQ}}+H_{A}$ corresponds to a configuration in which (i) adjacent spins are at right angles and (ii) each spin is along the $x$ or $z$ axis. There are many arrangements that satisfy these criteria and a few periodic states are illustrated in Fig. 1. Note that (a) has a two-layer unit cell while (b) and (c) have unit cells which are four layers in length. In the absence of a field, all these states are degenerate in energy. However, inclusion of $H_{Z}$ lifts the degeneracy. It is only necessary to consider $H$ in the positive octant $0 \leqslant \phi \leqslant 45^{\circ}$ in which case configuration (a), or rather the modification in finite field, turns out to have the lowest energy.

To obtain the energy and structure of the modified state (a), we need only specify two angles $\theta_{0}$ and $\theta_{1}$, since the unit cell for this structure contains only two

a)
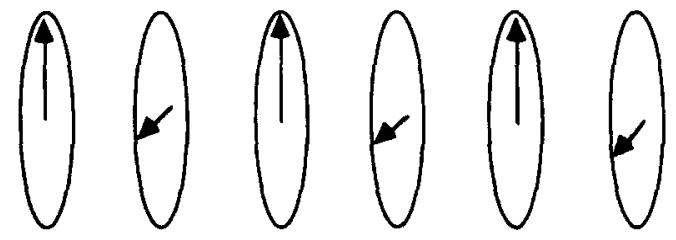

b)
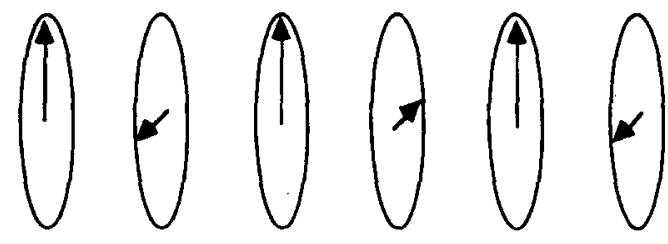

c)
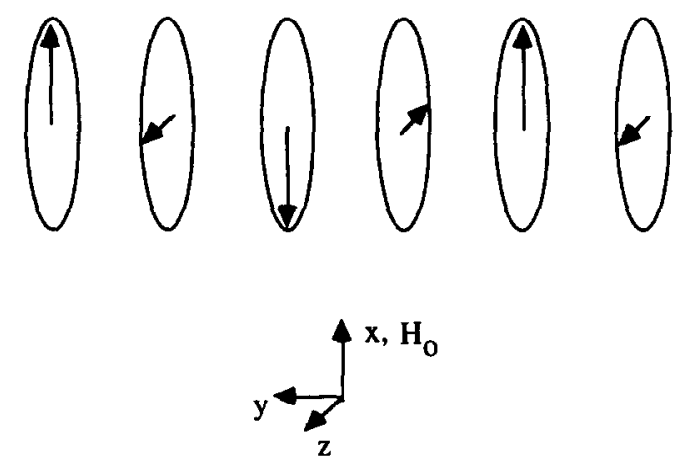

Fig. 1. Three possible eigenstates of $H_{\mathrm{BO}}+H_{A}$. Note that (a) has a unit cell of two layers and that (b) and (c) have unit cells of four layers. layers. We will call the resulting state the cross state since the spin vectors in neighbouring films are nearly perpendicular to each other and almost aligned with the $x$ and $z$ axes. The equations for minimum energy are

$$
\begin{aligned}
\partial H / \partial \theta_{0}= & K \sin 4 \theta_{0}+H \sin \left(\theta_{0}-\phi\right) \\
& -2 B \sin \left[2\left(\theta_{0}-\theta_{1}\right)\right]=0, \\
\partial H / \partial \theta_{1}= & K \sin 4 \theta_{1}+H \sin \left(\theta_{1}-\phi\right) \\
& +2 B \sin \left[2\left(\theta_{0}-\theta_{1}\right)\right]=0 .
\end{aligned}
$$

To see the implications of these two simultaneous equations for $\theta_{0}$ and $\theta_{1}$ we start with a small- $H$ expansion: $\theta_{0}=\epsilon_{0}$ and $\theta_{1}=\pi / 2-\epsilon_{1}$. Expanding equations (5) and (6) to terms linear in $H, \epsilon_{0}$ and $\epsilon_{1}$ we find

$$
\begin{aligned}
& \epsilon_{0}=H[(B+K) \sin \phi-B \cos \phi] / 4\left(K^{2}+2 B K\right), \\
& \epsilon_{1}=H[(B+K) \cos \phi-B \sin \phi] / 4\left(K^{2}+2 B K\right) .
\end{aligned}
$$

The energy of this state (per two-layer unit cell) is given by

$$
\begin{aligned}
E=- & 2 K-H(\cos \phi+\sin \phi)+2 B\left(\epsilon_{0}+\epsilon_{1}\right)^{2} \\
& +2 K\left(\epsilon_{0}^{2}+\epsilon_{1}^{2}\right)-H\left(\epsilon_{0} \sin \phi+\epsilon_{1} \cos \phi\right) .
\end{aligned}
$$

We note for later use that the last three terms on the right-hand side are proportional to $H^{2}$ which is the highest order that is retained in the small- $H$ expansion. For numerical illustrations we scale $B$ and $H$ in terms of the anisotropy constant, $b=B / K$ and $h=I I / K$. In the subsequent diagrams we take the value $b=0.2$. Such a value appears to be reasonable for high quality epitaxial growth of $\mathrm{Fe} / \mathrm{Cr} / \mathrm{Fe}$ sandwiches [1], however it would not be appropriate for permalloy where the anisotropy is much smaller [5].

Equations (7) and (8) determine the angles $\theta_{0}$ and $\theta_{1}$ (for small field) in terms of field strength $H$ and direction $\phi$ and we now explore some special case results. For $\phi=0$ (field along $x$ ) the first effect of the field is to turn $O_{1}$ away from the easy axis against the anisotropy field but toward the applied magnetic field (positive $\epsilon_{1}$ ). Because of the biquadratic coupling $\theta_{0}$ then turns slightly away from the easy axis and from the static field (negative $\epsilon_{0}$ ). It follows from equations (7) and (8) that $\left|\epsilon_{0}\right|<\epsilon_{1}$ so that $\theta_{1}$ turns through a larger angle than $\theta_{0}$. Physically the reason for this is that the system lowers its Zeeman energy when $\theta_{1}$ turns, but raises its energy when $\theta_{0}$ turns. For $\phi=45^{\circ}$ the spin orientations are symmetric about the field direction with both $\epsilon_{0}$ and $\epsilon_{1}$ positive.

We have carried out similar small-field expansions for modified configurations based on Fig. 1(b) and (c). Recall that both of these configurations have 
four-layer unit cells in contrast to the two-layer unit cell of the cross state discussed above. It turns out that the structure shown in Fig. 1(b) is metastable; it has an energy that is only slightly higher than the cross state discussed above. The helical-like structure of Fig. 1(c) has an energy that is quite a bit higher than the cross state. The numerical calculations confirm these conclusions and show that they are valid at higher fields as well.

There is an additional state which can be examined using the small angle approximation. For higher magnetic fields, it is reasonable to expect that the spins in all the layers will line up close to the direction of the external field. Subsequent numerical work indeed shows that this is a stable configuration. In this case the equilibrium equations are all identical. If we assume that $\theta$, the angle between the spin and the $x$ axis, is small then we obtain

$\theta=H \sin \phi /[H \cos \phi+4 K]$.

The energy of this state, per unit cell, is given by

$E=4 B-4 H \cos (\theta-\phi)-4 K\left(\sin ^{4} \theta+\cos ^{4} \theta\right)$.

The results above can be obtained without the additional assumption that $H$ is small. For example if $\phi$ is small and the spins are nearly lined up with the external field, then $\theta$ will also be small, independently of the size of $H$. Of course, it is unlikely that this will be the true ground state for very low fields.

Since the angular positions of the spins, $\theta$, is not necessarily in the same direction as the applied magnetic field, we will call this state an almost field aligned state (AFA). It seems reasonable to expect a transition from the cross state to the AFA state as the magnetic field is increased. We can obtain a critical field for such a transition by setting the energies of the two states equal to each other and solving for $H$. In order to compare the energy of the AFA state to that of the cross state we must first do a small- $H$ expansion for the AFA state energies so that both energies are valid to order $H^{2}$ only. When we do this we obtain the following expression for the critical field where the energies of the two states are equal

$H_{c}=\frac{-D \pm \sqrt{D^{2}-4 A C}}{2 A}$

where

$$
\begin{aligned}
A= & 4 B\left(\delta_{0}+\delta_{1}\right)^{2}+4 K\left(\delta_{0}^{2}+\delta_{1}^{2}\right) \\
& -2\left(\delta_{0} \sin \phi+\delta_{1} \cos \phi\right)+4 \delta \sin \phi, \\
D= & -2(\sin \phi-\cos \phi), \\
C= & -4 B,
\end{aligned}
$$

and $\delta=\theta / H, \delta_{0}=\epsilon_{0} / H, \delta_{1}=\epsilon_{1} / H$, these ratios being independent of $H$.

For an arbitrary value of the magnetic field, numerical solutions of the equilibrium equations, $\partial H / \partial \theta_{i}=0$, are required. We have explored these solutions using a method that has been applied previously to a number of problems [9-11]. For small $h$ the results confirm the expansions just discussed. As $h$ increases, at first the configuration remains qualitatively as sketched in Fig. 1(a) with the angles $\theta_{0}$ and $\theta_{1}$ slowly changing. We refer to this as a cross state. At a critical value $h_{c}$ there is a first order transition to the AFA state in which the spins are aligned but at an angle different from the external field. As $h$ increases above $h_{c}$ the angle between the spins and the field decreases until at very high field the spins are fully aligned along the field. The critical field $H_{c}$ given by equation (12) is within a few percent of the numerical results for $\phi<25^{\circ}$.

There are really two possible critical fields for the transition from the cross state to the AFA state. The first one corresponds to the field at which the energies of the cross state and the AFA state are equal. We refer to this as the thermal transition. Above this field the cross state continues to exist as a metastable state. Eventually, as the field is further increased the cross state is no longer stable, and we refer to this as the stability limit. It is not clear which transition would actually occur since a transition near the thermal critical field requires a mechanism to change the structure from a local minimum of the energy to a global minimum of the energy. We show in Fig. 2 the angular dependence of $h_{c}$, effectively the phase diagram.

The field dependence of the angles $\theta_{0}$ and $\theta_{1}$ for three field directions is shown in Fig. 3. For $\phi=0, \theta_{1}$ initially decreases linearly from $90^{\circ}$ while $\theta_{0}$ decreases linearly below $0^{\circ}$. The graphs are drawn for $b=B / K=0.2$. The abrupt transition to the AFA

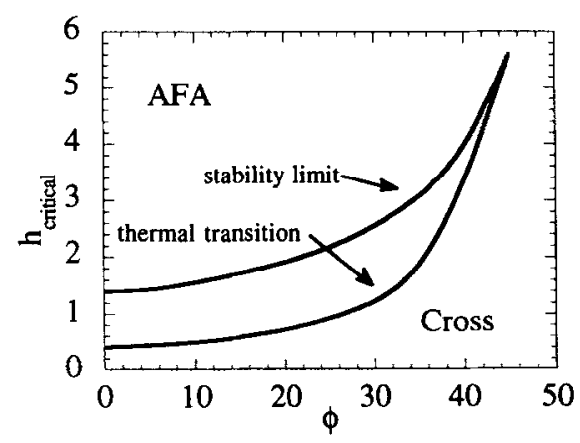

Fig. 2. Angular dependence of the critical fields $h_{c}$ for $b=B / K=0.2$. The curves separate the cross phase from the AFA phase. 

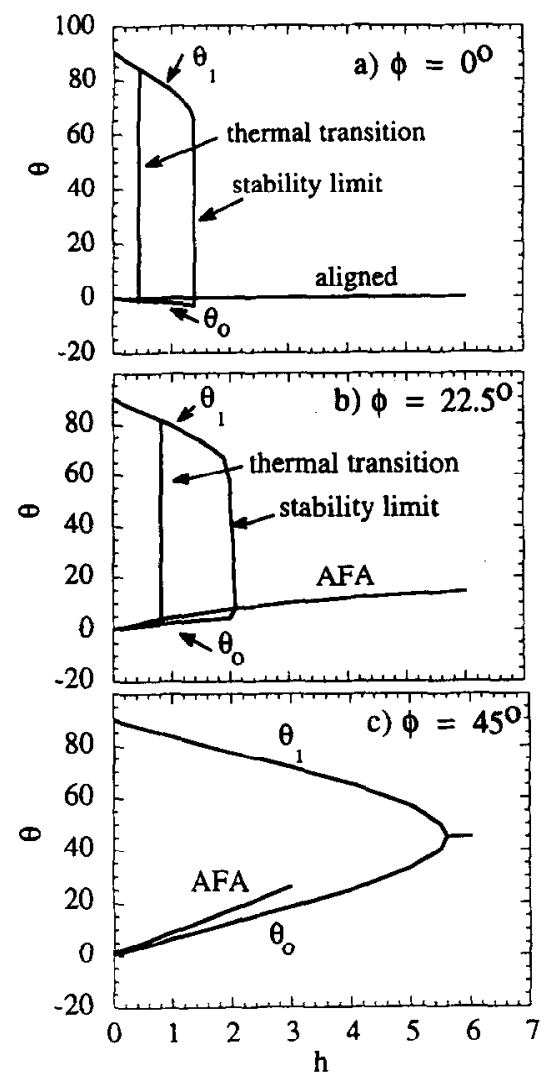

Fig. 3. Field dependence of the spin orientations $\theta_{0}$ and $\theta_{1}$ for $b=0.2$ and field directions (a) $\phi=0^{\circ}$, (b) $\phi=22.5^{\circ}$ and (c) $\phi=45^{\circ}$.

phase is seen for $\phi=0$ and also for $\phi=22.5^{\circ}$ in Fig. 3(b). The latter diagram is broadly similar to Fig. 3(a) except that the initial slope of $\theta_{0}$ is positive rather than negative. In addition, the misalignment between the spins and the applied field in the AFA state is seen clearly. Figure $3(\mathrm{c})$ shows the special case $\phi=45^{\circ}$ in which the curves for $\theta_{0}$ and $\theta_{1}$ are symmetric about the line $\theta=45^{\circ}$. Here, cxceptionally, the transition to the AFA state is continuous and the "AFA" state is in fact fully aligned. We note that in Fig. 3(a) and (b) the AFA state is metastable for fields below the critical fields and extends down to near $h=0$. In Fig. 3(c) the AFA state is metastable at low fields and is unstable for fields between 3 and 5.6. We did find another metastable state with a larger unit cell in this region but do not pursue this any further here.

A parameter that should be experimentally accessible is the magnetization. We show in Fig. 4 the field dependence of the components $S_{x}$ and $S_{z}$ for the same angles as those in Fig. 3 and for $b=0.2$ as in Fig. 3. For $\phi=0$, Fig. 4(a), the change in the cross state is that $S_{x}$ increases and $S_{z}$ decreases from the zero-field value of 0.5 . As seen from Fig. 3(a), the

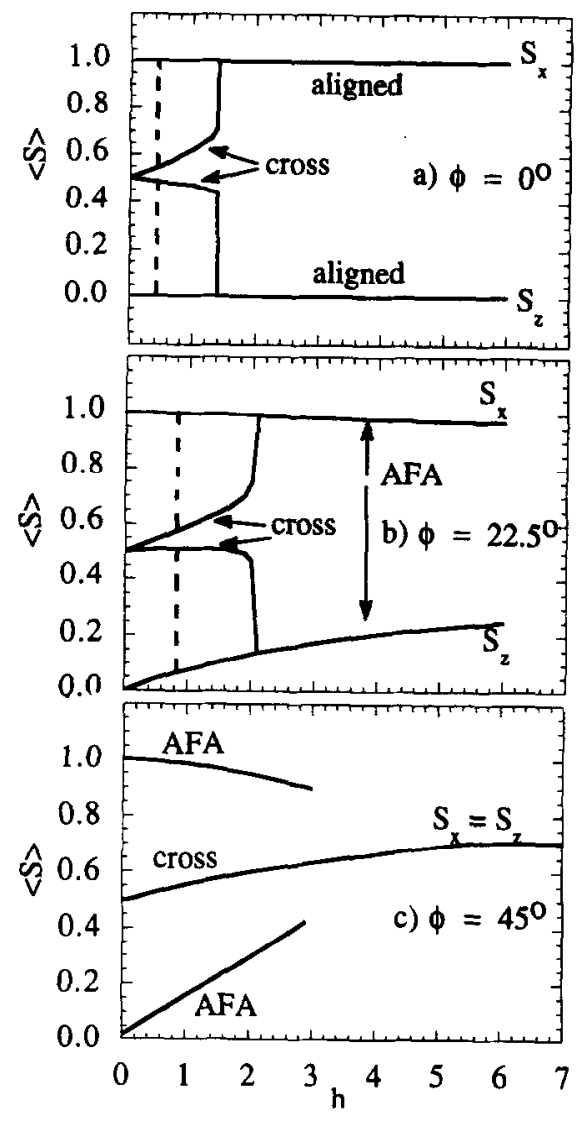

Fig. 4. Components $S_{x}$ and $S_{z}$ of the magnetization as a function of $h$ for $b=0.2$ and (a) $\phi=0^{\circ}$, (b) $\phi=22.5^{\circ}$ and (c) $\phi=45^{\circ}$. The thermal transition from the cross state to the AFA state is indicated by dotted lines. The stability threshold for the cross state is shown with solid vertical lines.

change with field of $\theta_{0}$ is very small compared with that of $\theta_{1}$ and consequently the field dependences of $S_{x}$ and $S_{z}$ are dominated by those due to $\theta_{1}$. Abrupt transition to the AFA state are clearly seen in Fig. 4(a). For $\phi=22.5^{\circ}$, Fig. 4(b), both $S_{x}$ and $S_{z}$ increase initially, in line with Fig. 3(b). The gradual alignment of $\theta_{0}$ and $\theta_{1}$ with the field direction seen in Fig. 3(b) is reflected in the gradual changes of $S_{x}$ and $S_{z}$ with field above $h_{r}$. Figure $4(\mathrm{c})$, for $\phi=45^{\circ}$, is consistent with Fig. 3(c) in that $S_{x}$ and $S_{z}$ are equal and increase with $h$ until the continuous transition into the fully aligned phase at $h_{c}$.

We note that the cross ground state discussed above can have a large magnetic moment perpendicular to the applied field if $\phi=0$. This is in sharp contrast to the state with the structure shown in Fig. 1(b) which has no transverse moment. In our calculations, the Fig. 1(b) structure has the higher energy, however it may be that dipolar interactions, neglected here, will favour the Fig. 1(b) structure. In this case the magnetization component along the field would be similar to the results 
obtained here but the magnetization transverse to the field would vanish.

In this work we have not included any ordinary linear nearest-neighbour (nn) exchange term $J \cos \left(\theta_{n}-\theta_{n-1}\right)$. For small $J$, such as might arise from a deviation of the $\mathrm{Cr}$ layer thicknesses from the design value, linear nn exchange does not produce any qualitative changes. The angle between neighbouring spins decreases for ferromagnetic exchange and increases for antiferromagnetic exchange. Large antiferromagnetic bilinear exchange, on the other hand, might stabilize helical configurations similar to those shown in Fig. I over those considered here and would therefore call for extensive further discussion.

We note that the situation discussed in this paper where the biquadratic exchange is larger than the bilinear exchange is relevant to multilayer systems. For example in $\mathrm{Co} / \mathrm{Cu}$ multilayers it has been shown that for thin $\mathrm{Cu}$ layers $(4-5 \AA)$ the biquadratic exchange is about 2-5 times larger than the bilinear exchange [5]. Other systems also show regions of parameter space where biquadratic exchange dominates. For example in NiFe/Ag multilayers $(10 \AA$ thick $\mathrm{Ag}$ ) at $T<100 \mathrm{~K}$ the biquadratic exchange is $50 \%$ larger than the bilinear [6]. Finally, we note that in $\mathrm{Fe} / \mathrm{Cr}$ systems biquadratic exchange dominates over bilinear at very low $\mathrm{Cr}$ thicknesses and also again at higher thicknesses [12].

We have made a start on a major extension, namely a study of systems with a finite number of layers. In this case, the outer films are only weakly exchanged coupled (since there is no coupling on one side), and the magnetic moment in outermost films will generally tend to point more closely along the applied field than the moments for films in the interior. Thus there will be a spatial distribution of angles and the magnetic "unit cell" can then be the entire structure. This material is beyond the scope of the present work and will be presented later.

Acknowledgements - REC was supported by US ARO Grant No. DAAH04-94-G-0253, and DAALO3-91-G-0229. ASC was supported by CNPq. We also acknowledge support of a NATO travel grant (REC, DRT) and a Royal Society/CNPq travel grant (DRT)

\section{REFERENCES}

1. M. Rürigh, R. Schäfer, A. Hubert, R. Mosler, J.A. Wolf, S. Demokritov \& P. Grünberg, Phys. Status Solidi (a) 125, 635 (1991).

2. B. Heinrich, Z. Celinski, J.F. Cochran, A.S. Arrott, K. Myrtle \& S.T. Purcell, Phys. Rev. B47, 5077 (1993).

3. S.T. Purcell, W. Folkerts, M.T. Johnson, N.W.R. McGee, K. Jager, J. aan de Stegge, W.B. Zeper \& W. Hoving, Phys. Rev. Lett. 67, 903 (1991).

4. M.E. Filipkowski, C.J. Gutierrez, J.J. Krebs \& G.A. Prinz, J. Appl. Phys. 73, 5963 (1993).

5. B. Rodmacq, K. Dumesnil, Ph. Mangin \& M. Hennion, Phys. Rev. B48, 3556 (1993).

6. J.F. Bobo, M. Piecuch \& E. Snoeck, J. Magn. Magn. Mater. 126, 440 (1993).

7. M. Maccio, M. G. Pini, P. Politi \& A. Rettori, Phys. Rev. B49, 3283 (1994).

8. See the review J.C. Slonczewski, J. Appl. Phys. 73, 5957 (1993).

9. R.E. Camley \& D.R. Tilley, Phys. Rev. B37, 3413 (1988).

10. A.S. Carriço \& R.E. Camley, Phys. Rev. B45, 13117 (1992).

11. For a review of work in this area see R.E. Camley \& R.L. Stamps, J. Phys. Condens. Matter. 5, 3727 (1993).

12. C. Daboo, R.J. Hicken \& J.A.C. Bland, Private communication. 